\title{
ROBUST VIDEO TEXT SEGMENTATION AND RECOGNITION WITH MULTIPLE HYPOTHESES
}

\author{
Jean-Marc Odobez and Datong Chen \\ IDIAP, Swizerland \\ \{odobez, chen\}@idiap.ch
}

\begin{abstract}
A method for segmenting and recognizing text embedded in video and images is proposed in this paper. In the method, multiple segmentation of the same text region is performed, thus producing multiple hypotheses of binary text images. The segmentation algorithm is stated as a statistical labeling and is based on a markov random field (MRF) model of the label map. Background regions in each hypothesis are then removed by performing a connected component analysis and by enforcing a more stringent constraint (called GCC) on the text characters grayscale values using a robust 1D-Median operator. Each text image hypothesis is then processed by an optical character recognition (OCR) software. The final result is then selected from the set of output strings. Results show that both the use of multiple hypotheses and the GCC significantly improve the results.
\end{abstract}

\section{INTRODUCTION}

Text recognition in video and images is now recognized as a key component in the development of advanced video and image annotation and retrieval systems. Text characters contained in video are of low resolution, of any grayscale value (not always white) and embedded in complex background even when the whole text string is well located. Thus, applying conventional OCR technology directly leads to poor recognition rate. Therfore, a large amount of work on text segmentation from complex background has been published in recent years. Lienhart [1] and Bunke [2] clustered text pixels from images using a common image segmentation or color clustering algorithm. Although these methods can somehow avoid the text location work, they are very sensitive to noise and character size. Most top down text segmentation methods are performed after text string is located in images. These methods assume that the grayscale distribution is bimodal and that characters correspond a priori to either the white part or the black one, but without providing a way of choosing the right one on-line. Great efforts are thus devoted to perform better binarization, combining global and local thresholding [3], or M-estimation [4], or simple smoothing [5]. However, these methods are unable to filter out background regions with similar grayscale value

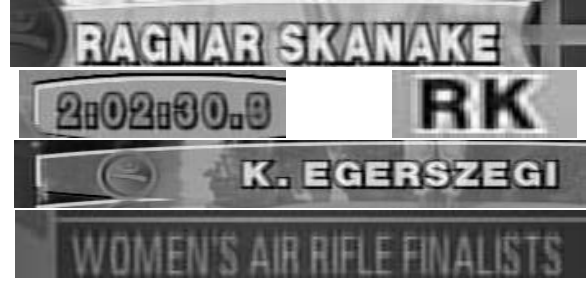

Fig. 1. Examples of located text in video

to characters. Text enhancement methods, if the character grayscale value is known, can help the binarization process [6]. However, without estimation of scales, the designed filters can not enhance character stroke with varying width [7]. In video, multi-frame enhancement [8] also can reduce the number of background regions, but only when text and background have different movements.

In this paper, we present a multi-hypotheses method based on MRF and on grayscale consistency constraint (GCC) to improve both the segmentation and recognition of embedded text with unknown grayscale value. We modelize the gray level distribution in the text images as a mixture of gaussians, and then assign each pixel to one of the gaussian layer. The assignment is based on prior of the contextual information, which is modeled by a MRF with online estimated coefficients. Each layer is considered as a candidate binary text image and is further processed. A GCC based connected component analysis module is first applied on each candidate to remove the background regions which have different shape or greyscale level than the text characters regions. The result is then forwarded to the OCR system as one segmentation hypothesis. By varying the number of gaussians, multiple hypotheses are provided to the OCR and the final result is selected from the set of outputs, leading to an improvement of the system's performances.

The next section presents in more detail our method. Commented results are given in Section 3 and Section 4 concludes the paper.

\section{DESCRIPTION OF THE METHOD}

Candidate text regions are first located using the method presented in [9]. In this method, text-like textures are 


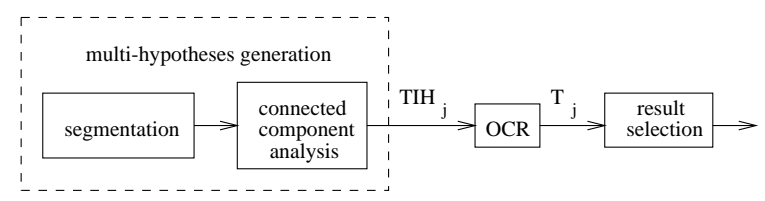

Fig. 2. Text recognition scheme

first detected by integrating horizontal and vertical edges, and further segmented into single line text candidates using baseline location. A support vector machine is then used to identify text regions from the candidates.

The text images provided by the text location step are in rectangles as those presented in figure 1. As we mentioned before, OCR software can not be applied directly. Indeed, experience shows that OCR performances are quite unstable, as already mentioned by others [1], and significantly rely on the segmentation quality, in the sense that errors made in the segmentation are directly forwarded to the OCR. In our case, we propose a softer scheme (see figure 2) in which multiple text layer candidates are provided to the OCR, delaying the hard decision, if any, after the OCR step. Our algorithm works as follows: first, text image hypotheses I I H $_{j}$ are generated, relying on a segmentation step followed by a connected component analysis; then hypotheses are processed by the OCR and the result is selected from the output strings $\left(T_{j}\right)_{j}$.

\subsection{The Gibbsian expectation maximization (GEM) segmentation method}

Let $S$ denote the set of sites $s$ (pixels), and $o$ the observation field $o=\left\{o_{s}, s \in S\right\}$, where $o_{s}$ corresponds to the grayscale value at site $s$. We model the image intensities in terms of the combination of $\mathrm{K}$ simple random processes, also referred to as layers. Each simple process is expected to represent regions of the image having similar gray levels, one of them being text. Thus, the segmentation consists in the mapping of pixels to processes. It is stated as a statistical labeling problem, where the goal is to find the label field $e=\left\{e_{s}, 1 \leq e_{s} \leq K, s \in S\right\}$ that best accounts for the observations, according to a given criterion. We perform a Maximum a-posteriori (MAP) optimization. Furthermore, to take into account the spatial correlation between assignment of pixels to layers, we model the label field as a markov random field (MRF). Due to the equivalence between MRF and Gibbs distribution $\left(\mathrm{p}(e)=\frac{1}{Z(V)} e^{-U_{1}^{V}(e)}\right)$ [10], the MAP optimization is equivalent to the minimization ${ }^{1}$ of an energy function $U(e, o)=U_{1}^{V}(e)+U_{2}^{\varphi}(e, o)$ where $U_{1}$ is equal to :

$$
\sum_{s \in S} V_{11}\left(e_{s}\right)+\sum_{<s, t>\in \mathcal{C}_{h v}} V_{12}^{h v}\left(e_{s}, e_{t}\right)+\sum_{<s, t>\in \mathcal{C}_{\text {diag }}} V_{12}^{d}\left(e_{s}, e_{t}\right)
$$

\footnotetext{
${ }^{1}$ with respect to $e$
}

and $\mathcal{C}_{h v}$ (resp. $\mathcal{C}_{\text {diag }}$ ) denotes the set of two neighbor pixels in the horizontal/vertical (resp. diagonal) direction. The $V$ are the (local) interaction potentials which expresses the prior on the labels. The energy term $U_{2}$ expresses the adequacy between observations and the labels. It is given by:

$$
U_{2}^{\varphi}(e, o)=\sum_{s \in S}\left(-\ln \mathrm{p}_{e_{s}}\left(o_{s}\right)\right) \quad\left(\varphi=\left(m_{i}, \sigma_{i}\right)_{i}\right)
$$

where the likelihood of a gray value $o$ at a site $s$ given a particular label $i$, is modeled by a gaussian, i.e. $\mathrm{p}_{i}\left(o_{s}\right)=$ $\mathcal{N}\left(m_{i}, \sigma_{i}\right)$. The use of off-line learned potential $V$ requires to know online the correspondence between learned labels and current ones. ${ }^{2}$ Instead, we propose the following algorithm to estimate all the parameters $\Theta=\left(\mu_{i}, \sigma_{i}, V\right)$ using an Expectation-Minimization procedure [11]. Recall that the E step involves the computation of :

$$
\mathbb{E}\left[\ln \mathrm{p}_{e o}^{\Theta} \mid o, \Theta^{n}\right]=\sum_{e} \ln \left(\mathrm{p}_{o \mid e}^{\Theta}(e, o) \mathrm{p}_{e}(e)\right) \mathrm{p}_{e \mid o}^{\Theta^{n}}(e, o)
$$

which is then maximized over $\Theta$. Two problems arise here. Firstly, this expectation on $\mathrm{p}_{e \mid O}^{\Theta^{n}}$ can not be computed explicitly nor directly. Instead, this law will be sampled using Monte Carlo methods with a Gibbs sampler, and the expectation will be approximated along the obtained Markov chain. Secondly, the joint log-likelihood probability $\mathrm{p}_{e o}^{\Theta}$ is not completely known, because of the presence of the uncomputable normalizing constant $Z(V)$ in the expression of $\mathrm{p}(e)$. To avoid this difficulty, we replace $\mathrm{p}(e)$ by its pseudo-likelihood $\mathrm{p}_{\mathrm{s}}(e)$ [12] defined from the conditional probabilities :

$$
\mathrm{p}_{\mathrm{s}}{ }^{V}(e) \doteq \prod_{s \in S} \mathrm{p}\left(e_{s} \mid e_{\mathcal{G}_{s}}\right)
$$

where $e_{\mathcal{G} s}$ represents the label in neighborhood of $s$. Using this new criterion, the maximization of the expectation (3) can be executed, providing new estimates of $\left(\mu_{i}, \sigma_{i}\right)$ and of $V$. The reader is referred to [12] for more details on the procedure that we have adapted to our need.

\subsection{GCC based connected component analysis}

After segmentation, for each label, a binary text image hypothesis is generated by assuming that this label corresponds to text and all other labels corresponds to background. In each hypothesis, the text regions are refined by using a simple connected component analysis. We keep only connected components that satisfy the following constraints: size is bigger than 5 pixels; width/height ratio is between 4.5 and 0.1 ; the width of the connected component is less than 2.1 of the height of the whole text region. The above step is especially useful in eliminating rather large and elongated non-text regions. However, it is

\footnotetext{
${ }^{2}$ Remind that text may be black or white or gray.
} 
unable to eliminate background regions with a gray value slightly different from that of characters but still belongs to the text layer/class. We thus developped another module to enforce a more stringent gray consistency among the connected components.

For a given layer, after the connected component analysis step, we estimate in a robust fashion the gray level mean $m^{*}$ and standard deviation $s t^{*}$ of the set of pixels $S_{r}$ belonging to the remaining regions. More precisely, we first compute :

$$
\begin{gathered}
m_{1}=\underset{m}{\operatorname{argmin}} M e d_{s \in S_{r}}\left|o_{s}-m\right|, r_{1}=\operatorname{Med}_{s \in S_{r}}\left|o_{s}-m_{1}\right| \\
\text { and } s t_{1}=1.48 \times\left(1+\frac{5}{\left|S_{r}\right|-1}\right) \times r_{1}
\end{gathered}
$$

where $M e d$ denotes a standard median operator. Valide pixels are then identified by checking that their gray value is within a given range of $m_{1}$, that is : $\frac{\left|o_{s}-m_{1}\right|}{s t_{1}}<k$ with $\mathrm{k}$ a given ratio (we use a value of 2). The Then the mean $m^{*}$ and standard deviation $s t^{*}$ are reestimated from the valid pixels only using the usual formula. Finally, a connected component is eliminated from the layer if more than $50 \%$ of its pixels have a gray level value different than the majority of pixels, that is, verify: $\frac{\left|o_{s}-m^{*}\right|}{s t^{*}}>k$. Figure 3 illustrates the result of this step.

\subsection{Text recognition}

The basic recognition of a text string from a single binary text image is performed using an OCR software. Indeed, in our method, we provide multiple text image hypotheses to the OCR and select the final result from the set of output strings based on a string confidence evaluation.

\subsubsection{The initial hypotheses generation:}

In the GEM segmentation method, if we have $\mathrm{K}$ different labels, we get $\mathrm{K}$ hypotheses. The right choice of $\mathrm{K}$ is another important and difficult issue. One general way to address the problem consists in checking whether the increase in model complexity really provides a better fit of the data. This can be done for instance by using the minimizing description length criterion. However, this information theoretic approach may not be appropriate for qualifying a good text segmentation. Therefore, we use a more conservative approach, by varying $\mathrm{K}$ from 2 to 4 , generating in this way nine text image hypotheses $T I H_{j}$.

\subsubsection{Result selection and confidence value :}

Each text image candidate $T I H_{j}$ is processed by the OCR software ${ }^{3}$ thus providing a string $T_{j}$. The final string result is the string $T_{j}$ that provides the largest confidence value $C V$ which is defined as :

$$
C V(T)=\sum_{i=0}^{l_{T}-1} f(T[i])+\sum_{i=1}^{l_{T}-1} g(T[i-1], T[i]) .
$$

\footnotetext{
${ }^{3}$ we use an open OCR toolkit (OpenRTK) from Expervision
}

\begin{tabular}{|c|c|c|c|c|c|}
\hline methods & K & Ext. & CRR & Prec. & WRR \\
\hline Otsu & 2 & $\mathbf{9 0 0 9}$ & $\mathbf{8 8 . 4 \%}$ & $\mathbf{9 3 . 8 \%}$ & $\mathbf{8 9 . 3 \%}$ \\
\hline GEM $^{*}$ & 2 & $\mathbf{9 0 8 1}$ & $\mathbf{8 8 . 7 \%}$ & $\mathbf{9 3 . 4 \%}$ & $\mathbf{9 0 . 8 \%}$ \\
GEM & 2 & $\mathbf{9 1 4 9}$ & $\mathbf{9 0 . 4 \%}$ & $\mathbf{9 4 . 5 \%}$ & $\mathbf{9 3 . 0 \%}$ \\
\hline GEM $^{*}$ & 3 & 9249 & $89.9 \%$ & $92.9 \%$ & $84.9 \%$ \\
GEM & 3 & 9196 & $90.1 \%$ & $93.7 \%$ & $86.6 \%$ \\
\hline GEM $^{*}$ & 4 & 9128 & $88.8 \%$ & $93.0 \%$ & $85.2 \%$ \\
GEM $^{*}$ & 4 & 9172 & $89.0 \%$ & $92.9 \%$ & $85.9 \%$ \\
\hline GEM $^{*}$ & $4 / 3 / 2$ & 9432 & $92.5 \%$ & $93.8 \%$ & $91.7 \%$ \\
GEM & $4 / 3 / 2$ & $\mathbf{9 4 6 0}$ & $\mathbf{9 2 . 7 \%}$ & $\mathbf{9 3 . 7 \%}$ & $\mathbf{9 3 . 1 \%}$ \\
\hline
\end{tabular}

Table 1. Recognition results in extracted characters (Ext.), character recognition rate (CRR), precision (Prec.) and word recognition rate (WRR). GEM* are the algorithms without using GCC.

where, $l_{T}$ is the length of string $T, g(x, y)$ is a simple bigram language model defined as :

$$
\begin{aligned}
& g(x, y)=\left\{\begin{aligned}
-4 & \text { if } x=\text { lower case, } y=\text { upper case } \\
0 & \text { otherwise }
\end{aligned}\right. \\
& \text { and } f(x)=\left\{\begin{aligned}
4 & \text { if } x=\text { upper case } \\
2 & \text { if } x=\text { lower case } \\
0 & \text { if } x=i, I, l \\
-1 & \text { otherwise }
\end{aligned}\right.
\end{aligned}
$$

Some characters (i, I, l) and lower case characters are given lower weights because background noise is more often recognized as these characters.

\section{EXPERIMENTS}

The whole scheme was tested on text regions located and extracted from one hour of sports video provided by the $\mathrm{BBC}$, using the algorithm presented in [9]. It correctly located $98.7 \%$ text regions while providing $0.38 \%$ false alarms. We randomly selected 1208 images from the extracted text regions, mainly by time sub-sampling, providing a database of 9562 characters or 867 words. Figure 1 shows some examples. Text characters are embedded in complex background with JPEG compression noise, and the grayscale value of characters is not always the highest. Performances are evaluated using character recognition rates (CRR) and precision rates (Prec) that are computed on a ground truth basis as :

$$
C R R=\frac{N_{r}}{N} \text { and } C R R=\frac{N_{e}}{N}
$$

$N$ is the true total number of characters, $N_{r}$ is the number of correctly recognized characters and $N_{e}$ is the total number of extracted characters. Additionally, we compute the word recognition rate to get an idea of the coherency of character recognition within one solution. For each text image, we 
count the words from the ground truth of that image that appear in the string result. Results are listed in table 1.

Considering the binarization case $(\mathrm{K}=2)$, we can see that the GEM algorithm provides slightly better results than the Otsu algorithm. It is due to the GEM adaptability, which, by learning the local spatial properties of the grayscale distribution, is noise adaptive and is able to better avoid over segmentation. However, the gain is not so significant and can be explained by the fact that the GEM algorithm mainly improves the shape of the characters. This improvement is somehow cancelled out by the OCR which is indeed able to cope with shape noise. However, the use of the GCC improves the results a lot. It can be explained similarly, by the fact that background regions that are not removed greatly affects the OCR performances. The GCC step succeeds well in this task by keeping the character components and removing the background regions.

Similarly, the last rows of table 1 list the results obtained by generating 9 hypotheses (using $K=2$ to 4 ). They show that even with our simple confidence criteria, and without the GCC, the improvement is very significant, with a reduction of $35 \%$ with respect to the standard Otsu algorithm of the character error rate. Together with the GCC, the reduction in both character and word error rate with respect to the Otsu algorithm is about $36 \%$.

The word recognition can be improved by keeping the best two or best three strings with respect to the confidence level. We obtain a $97.5 \%$ word recognition rate using the highest two hypotheses and a $97.9 \%$ using three hypotheses in GEM, which significantly improve the result of $93.1 \%$ obtained when keeping only the best hypothesis. This can yield better text searching results by offering more precise keywords in image and video indexing and retrieval system. Also, this means that there is room for improvement using a better selection scheme.

\section{CONCLUSION}

In this paper, we proposed a method for segmenting and recognizing embedded text of any grayscale value in image and video based on MRF, gray scale constraints and a multiple hypotheses scheme. Experiments have shown that the use of these multiple hypotheses and/or of the gray scale constraint significantly improve the results with respect to Otsu algorithm.

\section{ACKNOWLEGEMENT}

This work has been performed partially with in the framework of the "Combined Image and Word Spotting (CIMWOS)" project granted by the European IST Programme. a)

b)

c)

d)

e)

Fig. 3. Applying grayscale consistency : a) original image b) text layer (GEM K=3) c) same as b), after the connected component analysis d) text layer (Otsu), e) same as d), after the connected component analysis, f) same as c), after the gray level consistency step.

\section{REFERENCES}

[1] R. Lienhart, "Automatic text recognition in digital videos," in Proc. SPIE, Image and Video Processing $I V$, Jan. 1996, pp. 2666-2675.

[2] K. Sobottka, H. Bunke, and H. Kronenberg, "Identification of text on colored book and journal covers," in ICDAR, 1999, pp. 57-63.

[3] H. Kamada amd K. Fujimoto, "High-speed, highaccuracy binrization method for recognizing text in images of low spatial resolutions," in ICDAR, Sept. 1999, pp. 139-142.

[4] O. Hori, "A video text extraction method for character recogntion," in ICDAR, Sept. 1999, pp. 25-28.

[5] V. Wu, R. Manmatha, and E. M. Riseman, "Finding text in images," in Proc. ACM Int. Conf. Digital Libraries, 1997, pp. 23-26.

[6] T. Sato, T. Kanade, E. K. Hughes, and M. A. Smith, "Video ocr for digital news archives," in IEEE Workshop on Content Based Access of Image and Video Databases, Jan. 1998, Bombay.

[7] D. Chen, K. Shearer, and H. Bourlard, "Text enhancement with asymmetric filter for video ocr," in Proc. of the 11th Int. Conf. on Image Analysis and Processing, Sept. 2001, pp. 192-198.

[8] H. Li and D. Doermann, "Text enhancement in digital video using multiple frame integration," in $A C M$ Multimedia, 1999, pp. 385-395. 
[9] D. Chen, H. Bourlard, and J-Ph. Thiran, "Text identification in complex background using svm," in Proc. of the Int. Conf. on computer vision and pattern recognition, Dec. 2001, vol. 2, pp. 621-626.

[10] S. Geman and D. Geman, "Stochastic relaxation, Gibbs distributions and the Bayesian restoration of images," PAMI, vol. Vol.6, No.6, pp. 721-741, Nov. 1984.

[11] A. Dempster, N. Laird, and D. Rubin, "Maximumlikelihood from incomplete data via the em algorithm," Royal Statistical Society, vol. B-39, pp. 1-38, 1977.

[12] B. Chalmond, "Image restoration using an estimated Markov model," Signal Processing, vol. 15, no. 2, pp. 115-129, Sept. 1988. 